Nadir do Nascimento Nogueira 1

\title{
Mudanças na concentração plasmática de zinco e ácido fólico em adolescentes grávidas submetidas a diferentes esquemas de suplementação
}

\author{
Changes in plasma zinc and folic acid \\ concentrations in pregnant adolescents submitted \\ to different supplementation regimens
}

Joaquim Vaz Parente 2

Sílvia Maria Franciscato Cozzolino 3

\footnotetext{
1 Departamento de Nutrição, Centro de Ciências da Saúde, Universidade Federal do Piauí. Campus Universitário do Ininga, Teresina, $P I$ 64049-550, Brasil. nadirn@uol.com.br 2 Departamento MaternoInfantil, Universidade Federal do Piauí. Av. Frei Serafim 2280, Teresina, $P I$ 64000-010, Brasil.

3 Faculdade de Ciências Farmacêuticas, Universidade de São Paulo. Av. Prof. Lineu Prestes 580, São Paulo, SP 05315-970, Brasil.
}

\begin{abstract}
Zinc and folic acid nutritional status was evaluated in 74 low-income pregnant adolescents ranging from 13 to 18 years of age who received prenatal care at the Evangelina Rosa Maternity Hospital in Teresina, Piaui State, Brazil. In order to evaluate the effects of different supplementation regimens on nutritional status, the adolescents were distributed into five groups. Groups I and II received equal amounts of folic acid (250 $\mu$ g) and different doses of iron (ferrous sulfate), 120 and $80 \mathrm{mg}$, respectively. Groups III and IV received equal amounts of folic

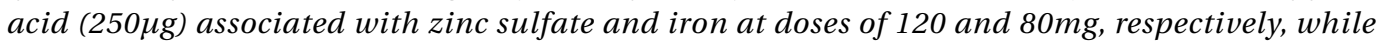
group $V$ received only $120 \mathrm{mg}$ of iron (routine dosage). There was a reduction in the zinc plasma concentration, and this decline was significant only in those groups which did not receive zinc supplementation. In relation to combination iron/folic acid and iron/folic acid/zinc, an excellent response was observed for folic acid, and this effect was larger in the groups that received folic acid combined with zinc, suggesting a possible role for zinc in folic acid metabolism.
\end{abstract}

Key words Pregnancy in Adolescence; Iron; Folic Acid; Zinc; Nutritional Status

Resumo O estado nutricional do zinco e ácido fólico foi avaliado em 74 adolescentes grávidas, de baixa renda, entre 13 e 18 anos, atendidas no pré-natal na Maternidade Dona Evangelina Rosa, Teresina, Piauí, Brasil. Para avaliar os diferentes esquemas de suplementação no estado nutricional das adolescentes, estas foram aleatoriamente distribuídas em cinco grupos: Os grupos I e II receberam quantidades iguais de ácido fólico (250 $\mu \mathrm{g}$ ) e diferentes concentrações em ferro (sulfato ferroso) 120 e $80 \mathrm{mg}$, respectivamente. Os grupos III e IV receberam quantidades iguais de ácido fólico (250 $\mathrm{\mu g}$ ) associado ao sulfato de zinco (5mg) e ferro nas concentrações de 120 e $80 \mathrm{mg}$, respectivamente e o grupo $\mathrm{V}$, apenas ferro, $120 \mathrm{mg}$ (rotina). Os resultados quanto ao zinco mostraram uma redução na sua concentração plasmática, sendo esse declínio significativo apenas nos grupos que não receberam o mineral. Quanto ao uso associado de ferro/ácido fólico e ferro/ácido fólico/zinco, verifica-se excelente resposta no estado nutricional referente ao ácido fólico, sendo esse efeito mais expressivo nos grupos que receberam ácido fólico associado ao zinco, sugerindo a possível participação do zinco no aproveitamento da vitamina.

Palavras-chave Gravidez na Adolescência; Ferro; Ácido Fólico; Zinco; Estado Nutricional 


\section{Introdução}

Em todo o mundo, as mulheres grávidas constituem um grupo especialmente vulnerável à ocorrência da anemia ferropriva, devido à ingestão insuficiente de ferro na dieta e ao aumento das necessidades metabólicas. No entanto, existem poucos dados sobre os efeitos da suplementação com esse mineral durante o pré-natal de mulheres de países em desenvolvimento.

Como medida de intervenção, a suplementação associada de ferro e ácido fólico constitui a principal estratégia para o controle da anemia na gravidez. Nesse sentido, mecanismos de aplicação dessa estratégia têm sido revisados nos últimos anos, afim de se alcançar o controle efetivo da anemia deste grupo populacional (Diego et al., 1997; Zavaleta et al., 2000).

Embora o foco da maioria dos estudos sobre suplementação de minerais durante a gravidez seja o ferro, a ingestão alimentar de outros minerais tais como o zinco é também reduzida em áreas deficientes em ferro.

Nesse contexto, e levando em conta o ciclo reprodutivo, a adolescente grávida é considerada como nutricionalmente de risco, tendo em vista que a maioria delas ainda se encontra em processo de crescimento e desenvolvimento, além da necessidade de uma demanda extra sobre o seu organismo, para o crescimento e desenvolvimento do feto (Coates, 1993).

Ferro, zinco e ácido fólico são nutrientes essenciais que freqüentemente estão deficientes nas dietas de adolescentes. O ferro e o ácido fólico são necessários para o desenvolvimento normal do sistema hematopoiético, enquanto o zinco desempenha importante função na manutenção da integridade estrutural e na regulação da expressão gênica (Institute of Medicine, 2001). Goldenber et al., (1995), sugerem que suplementos contendo o zinco melhoram as condições da gravidez. Nesse contexto, evidências têm apoiado a hipótese de que o nível de zinco materno pode ser um preditor de complicações na gravidez (Nichols et al., 1992).

O Instituto de Perinatologia Social do Piauí - Maternidade Dona Evangelina Rosa (MDER), Nordeste, Brasil, local de desenvolvimento deste estudo, tem uma demanda média mensal de 300 adolescentes grávidas. No entanto, apesar do número de atendimento, o serviço não dispõe de dados sobre a condição nutricional da gestante, de uma maneira geral, e particularmente no que se refere a alguns micronutrientes de relevante papel durante o período gestacional. Levando em consideração essa carência de dados, em nível local, e a escassez na li- teratura de informações referentes ao uso de suplementos contendo ferro, zinco e ácido fólico por adolescentes grávidas e de seus efeitos, desenvolvemos esta pesquisa objetivando avaliar, dentre outros parâmetros, alterações plasmáticas nas concentrações de zinco e ácido fólico em adolescentes grávidas que fizeram uso de diferentes esquemas de suplementação.

\section{Casuística e métodos}

A população estudada foi constituída inicialmente por 114 adolescentes grávidas, com idade entre 13 e 18 anos, que eram atendidas no Instituto de Perinatologia Social do Piauí MDER, e se encontravam no início da pesquisa entre 16 e 20 semanas de gravidez. A idade gestacional foi calculada de acordo com a data da última menstruação, e na incerteza desta, a data era confirmada pelo exame de ultra-sonografia.

Neste estudo, de natureza longitudinal, prospectivo e de intervenção, algumas perdas ocorreram, onde os principais motivos foram: abandono do pré-natal ou mudança para outra unidade de saúde - 22 casos $(56,4 \%)$; oito por intolerância aos medicamentos (20,5\%); três abortos espontâneos $(7,7 \%)$; três por erro na idade gestacional $(7,7 \%)$ e igual valor por parto prematuro $(7,7 \%)$ tendo o estudo sido concluído com uma amostra de 74 adolescentes grávidas.

Para seleção da amostra, os seguintes critérios de elegibilidade foram utilizados: a adolescente encontrar-se entre 16 e 20 semanas de gravidez, não apresentar complicações obstétricas ou médicas; ocorrência de aborto num período mínimo de seis meses antes da pesquisa; feito uso de qualquer hematínico e contraceptivo oral, no período de seis meses imediatamente anterior à concepção; fazer uso de drogas, fumo e álcool, e uso de dietas especiais (vegetariana, macrobiótica, etc.), variáveis estas, consideradas confundidoras, quando se pretende realizar estudos com esse propósito.

Após aprovação do protocolo da pesquisa pelo comitê de ética da Instituição, e as adolescentes estarem cientes e concordantes com o estudo, por meio de sorteio as mesmas foram aleatoriamente distribuídas em cinco grupos, recebendo o seguinte esquema terapêutico: Grupo I: $120 \mathrm{mg}$ de ferro e $250 \mu \mathrm{g}$ de ácido fólico; II: $80 \mathrm{mg}$ de ferro e $250 \mu \mathrm{g}$ de ácido fólico; III: $120 \mathrm{mg}$ de ferro e $250 \mu \mathrm{g}$ de ácido fólico associado a $5 \mathrm{mg}$ de zinco; IV: $80 \mathrm{mg}$ de ferro, $250 \mu \mathrm{g}$ de ácido fólico associado a $5 \mathrm{mg}$ de zinco e Grupo V: $120 \mathrm{mg}$ de ferro (rotina local). A suplementação foi iniciada com as adolescentes entre $16 \mathrm{e}$ 
20 semanas de gravidez até o final da gestação, onde apenas os pesquisadores sabiam a que grupo cada gestante pertencia. O composto ferro era oferecido na forma de sulfato ferroso $\left(\mathrm{Fe}_{2}, \mathrm{SO}_{4}\right)$, administrado oralmente, em duas (80mg) ou três vezes ao dia (120mg). Quanto ao ácido fólico, este foi fornecido na forma de pteroilmonoglutamato, isolado ou associado ao sulfato de zinco $\left(\mathrm{ZnSO}_{4}\right)$ e tomado em dose única, associado ou não ao ferro.

Para efeito de controle e avaliação da intervenção, as adolescentes tinham mensalmente agendamento com o médico e com a responsável pela pesquisa, até o período do parto. $\mathrm{Na}$ consulta, era realizado o controle do uso dos medicamentos, pela contagem dos comprimidos restantes na cartela, e por meio de informação prestada pela paciente. Em seguida, era feita a entrega dos medicamentos do mês subseqüente. Na oportunidade, realizavam-se também tomadas de peso e altura e eram investigados eventuais problemas com a alimentação e rejeição aos medicamentos, bem como de qualquer tipo de intercorrência ligada à gravidez. Considerando: (1) possíveis reações adversas aos medicamentos; (2) possibilidade de eventuais esquecimentos no uso dos mesmos e (3) falta à consulta, adotou-se como critério de permanência no estudo, o prazo total de 15 dias, de forma descontínua, sem uso dos medicamentos.

Para a realização das avaliações hematológica e bioquímica referentes aos nutrientes em estudo, foram coletados $12 \mathrm{ml}$ de sangue venoso, em dois momentos, com as pacientes em jejum de no mínimo 10 horas: uma no período pré-intervenção, estando as adolescentes entre 16 a 20 semanas de gravidez, e a outra no pósintervenção, com as mesmas entre 37 a 38 semanas de gravidez.

As determinações das concentrações plasmáticas de zinco e de ácido fólico nas amostras foram realizadas segundo os métodos da espectrofotometria de absorção atômica e radioimunoensaio-agente marcador-Iodo 125 , respectivamente.

A análise estatística dos dados acerca da homogeneidade dos grupos para as variáveis investigadas foi realizada aplicando-se o teste de variância (ANOVA). Para se obter uma maior estabilidade das variáveis, utilizou-se o logarítimo dos dados, sem prejuízo para a significância dos resultados. Para efeito de comparação de valores, indivíduo a indivíduo, foi utilizado o teste "T-Student" pareado e o não paramétrico de Wilcoxon (Noether, 1980), com significância definida com $\mathrm{p}<0,05$.

\section{Resultados}

Em termos de caracterização do grupo de adolescentes estudado, a média de idade das mesmas foi de 16 anos; idade da menarca de 12,5 anos e $100 \%$ eram primíparas. Quanto ao nível de escolaridade, $92 \%$ delas tinham o 1 o grau incompleto. No que diz respeito à condição nutricional, de acordo com o índice de massa corpórea (IMC), 65,3\% das adolescentes eram eutróficas $(20-24,99), 28 \%$ apresentavam baixo peso $(<20)$ e $6,7 \%$ tinham sobrepeso $(25-29,99)$.

Com relação aos micronutrientes pesquisados, a média das concentrações plasmáticas de zinco da população pesquisada, no período pré-intervenção foi de $59,33 \mu \mathrm{g} / \mathrm{mL}(9,08 \mu \mathrm{mol} / \mathrm{L})$, estando portanto abaixo do ponto de corte $(68,9 \mu \mathrm{g} / \mathrm{dL}$ ou $10,4 \mu \mathrm{mol} / \mathrm{L})$, para esse período gestacional (Hambidge \& Droegmuller, 1974), comportamento semelhante ao observado entre os 5 grupos estudados. No total de adolescentes estudadas, a prevalência de baixos valores do mineral nesse período foi de $79 \%$. No pósintervenção, observa-se nos diferentes grupos, um declínio na concentração plasmática de zinco, porém com significado estatístico somente para os grupos I $(49,38 \pm 8,8)$ e II $(45,80 \pm 12,0)$ (Tabela 1).

A Tabela 2 traz as médias das concentrações de ácido fólico plasmático, nos períodos pré e pós-intervenção. A suplementação com os nutrientes em estudo elevou a concentração plasmática da vitamina nos cinco grupos de intervenção. No entanto, esse aumento foi estatisticamente significativo apenas nos grupos III $(15,35 \pm 10,0)$ e IV $(14,56 \pm 4,0)$, e diminuição nos valores do grupo $V(5,32 \pm 2,0)$, porém sem significado estatístico. Na amostra pesquisada antes da intervenção, a prevalência de baixos valores plamáticos da vitamina $(<6 \mathrm{ng} / \mathrm{mL})$ foi de $37 \%$.

\section{Discussão}

Poucos são os dados disponíveis no mundo e particularmente no Brasil, sobre anemia por deficiência de ferro e ácido fólico em adolescentes grávidas. Em nosso meio, a anemia nesse grupo alcança valores elevados como mostram os dados de prevalência encontrados por Salzano et al., (1980) e Szarfarc (1983), de 40\% e $37,5 \%$, respectivamente, semelhantes, portanto, à prevalência encontrada neste estudo 35\% (Nogueira et al. 2001), porém contrários aos verificados por Fujimori et al. (1999, 2000), ficando em torno de $14 \%$.

Com relação ao zinco e ácido fólico, antes que se faça qualquer discussão acerca das alte- 
Tabela 1

Valores médios das concentrações de zinco plasmático das adolescentes, segundo os grupos pesquisados, nos períodos pré e pós-intervenção. Instituto de Perinatologia Social do Piauí, Maternidade Dona Evangelina Rosa, Teresina, Piauí, Brasil, 1996.

\begin{tabular}{llccc}
\hline Grupos & $\begin{array}{c}\text { Pré-intervenção } \\
\text { Zinco }(\mu \mathrm{g} / \mathrm{mL})\end{array}$ & $\begin{array}{c}\text { Intervalo com 95\% } \\
\text { de confiança }\end{array}$ & $\begin{array}{c}\text { Pós-intervenção } \\
\text { Zinco }(\mu \mathrm{g} / \mathrm{mL})\end{array}$ & $\begin{array}{c}\text { Intervalo com 95\% } \\
\text { de confiança }\end{array}$ \\
\hline Grupo I & $62,13 \pm 12(15)^{\star}$ & $56,00-68,26$ & $49,38 \pm 8,8(15)$ & $44,90-53,88$ \\
Grupo II & $58,96 \pm 13(13)$ & $51,88-66,04$ & $45,80 \pm 12,0(12)$ & $32,97-51,59$ \\
Grupo III & $57,05 \pm 11(16)$ & $51,60-62,50$ & $49,34 \pm 9,9(14)$ & $33,77-52,59$ \\
Grupo IV & $59,55 \pm 15(17)$ & $52,16-66,94$ & $50,21 \pm 12,0(17)$ & $44,49-55,93$ \\
Grupo V & $58,96 \pm 14(12)$ & $42,94-65,92$ & $48,52 \pm 11,6(13)$ & $42,17-54,87$ \\
\hline
\end{tabular}

* Número de casos.

Tabela 2

Médias das concentrações de ácido fólico plasmático de adolescentes grávidas, de acordo com os grupos, antes e depois da intervenção. Instituto de Perinatologia Social do Piauí, Maternidade Dona Evangelina Rosa, Teresina, Piauí, Brasil, 1996.

\begin{tabular}{lcccc}
\hline Grupos & $\begin{array}{c}\text { Pré-intervenção } \\
\boldsymbol{\mu g} / \mathbf{m L}\end{array}$ & $\begin{array}{c}\text { Intervalo com 95\% } \\
\text { de confiança }\end{array}$ & $\begin{array}{c}\text { Pós-intervenção } \\
\boldsymbol{\mu g} / \mathbf{m L}\end{array}$ & $\begin{array}{c}\text { Intervalo com 95\% } \\
\text { de confiança }\end{array}$ \\
\hline Grupo I & $10,0 \pm 5,4(13)^{\star}$ & $7,1-12,9$ & $15,7 \pm 11,3(12)$ & $9,3-22,0$ \\
Grupo II & $8,3 \pm 3,3(13)$ & $6,5-10,0$ & $12,7 \pm 6,7(11)$ & $8,6-16,8$ \\
Grupo III & $7,4 \pm 5,4(12)$ & $5,9-8,9$ & $15,3 \pm 10,0(14)$ & $10,0-20,5$ \\
Grupo IV & $7,7 \pm 4,2(14)$ & $5,5-9,9$ & $14,6 \pm 4,0(13)$ & $12,4-26,9$ \\
Grupo V & $8,0 \pm 6,7(9)$ & $3,6-12,4$ & $5,3 \pm 2,0(9)$ & $4,0-6,6$ \\
\hline
\end{tabular}

* Número de casos.

rações plasmáticas desses nutrientes, é oportuno destacar que a gravidez é caracterizada por mudanças fisiológicas e metabólicas que alteram parâmetros bioquímicos e hematológicos maternos, resultando em diminuição ou aumento dos mesmos, quando comparados a valores normais da mulher não grávida (Knight et al., 1994).

No que diz respeito ao zinco, analisando-se de forma comparativa os diferentes esquemas de suplementação empregados nos dois períodos deste estudo, observa-se no período pósintervenção um declínio na concentração plasmática do zinco das adolescentes dos cinco grupos pesquisados, sendo esta queda significante ( $p<0,05)$ somente para os grupos I e II, cujas adolescentes não receberam esse mineral. Essa resposta pode, ao contrário de outros estudos (Hunt et al., 1985; Keen \& Hurlet, 1989), ter sido influenciada pelo acréscimo do mesmo, o que vem confirmar o quadro de deficiência verificado no período pré-intervenção (79\%).
É importante ressaltar que durante a gravidez a concentração de zinco plasmático diminui de $20 \%$ a $30 \%$, a partir do terceiro mês de gravidez, quando comparada a valores de mulheres não grávidas (Kirksey et al., 1994; Zimmerman et al., 1984). Vários mecanismos têm sido propostos para explicar esse declínio. Um consenso existe de que essa redução seja devido à expansão do volume plasmático (Swanson \& King, 1983; Tuttle et al., 1985).

A deficiência alimentar de folato também é freqüente entre as mulheres grávidas. Mais de um terço das gestantes no mundo são deficientes em ácido fólico. A incidência é oito vezes maior em gestações múltiplas, e parece ser aumentada em adolescentes grávidas (Campbell, 1995). Da mesma forma que ocorre com o zinco, a concentração plasmática de ácido fólico cai durante a gravidez, devido à expansão do volume plasmático, e dessa maneira deverá ser considerada como fisiológica. Para o período de 16 a 20 semanas de gravidez, foram encon- 
tradas concentrações médias de $8,3 \mu \mathrm{g} / \mathrm{mL}$, superiores portanto, às verificadas por Hall

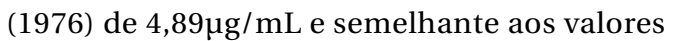
de Baily (1980) $-8,2 \mu \mathrm{g} / \mathrm{mL}$.

Analisando-se o comportamento do ácido fólico, entre os grupos, no período pós-intervenção, o que se verifica é um aumento significante nas concentrações plasmáticas da vitamina, somente nos grupos III $(15,35 \pm 10,0) \mathrm{e}$ IV $(14,56 \pm 4,0)$ e diminuição nos valores do grupo $V(5,32 \pm 2,0)$, porém sem significado estatístico. É importante ressaltar que o grupo $\mathrm{V}$ não recebeu ácido fólico e os III e IV além de ferro e folato receberam também $5 \mathrm{mg}$ de zinco.

O estudo de Tamura et al. (1978), revela que a absorção do poliglutamil folato encontravase reduzida em homens deficientes em zinco. O mecanismo proposto para essa redução foi posteriormente apresentado por outros pesquisadores que sugeriram ser decorrente da redução na atividade da conjugase folato (Zn dependente), necessária ao processo de absorção da vitamina (Kaiser et al., 1981).
Considerando-se os dados apresentados, fica evidente que as adolescentes grávidas envolvidas neste estudo encontravam-se com o estado nutricional comprometido quanto ao zinco e ácido fólico. Verifica-se ainda que o uso de suplementos contendo ferro associado ao ácido fólico e ferro/ácido fólico/zinco, apesar de não ter provocado em todos os grupos de intervenção mudanças no estado nutricional das adolescentes, com significado estatístico, as estratégias representaram importância clínica, haja visto as reduções observadas nas prevalências de anemia e de baixos valores nas concentrações plasmáticas de zinco e de ácido fólico, resultados estes apoiados por Zavaleta et al. (2000).

Sugere-se com base nesses dados, a realização de outros estudos com adolescentes grávidas, para melhor definição acerca das concentrações e freqüência de uso dos suplementos contendo ferro, ácido fólico e zinco que venham a atender às necessidades desse grupo. Acresce-se ainda, que essa estratégia de intervenção deverá estar associada a um bom acompanhamento pré-natal e ao envolvimento de uma equipe multidisciplinar.

\section{Agradecimentos}

Às adolescentes, pela participação e envolvimento com o estudo. Aos médicos - Dr. Vicente de Paula Canapum, Dra. Márcia Santana J. Martins e Carolina dos Santos Oliveira pelo total apoio à condução desta pesquisa, e pela valiosa colaboração das alunas de iniciação científica Dilina do Nascimento Marreiro e Mária Andreia Moura Fé. 


\section{Referências}

BAILY, L. B., 1980. Folacin and iron status in low income pregnant adolescents and mature women. American Journal of Clinical Nutrition, 33:19972001.

COATES, V., 1993. Necessidades nutricionais na gravidez. In: Medicina do Adolescente (G. Beznos, V. Coates \& L. A. Françoso, org.), pp. 263-267, São Paulo: Sarvier.

CAMPBELL, B. A., 1995. Megaloblastic anemia in pregnancy. Clinical Obstetric and Gynecology, 38:455-462.

DIEGO, C.; RODRIGO, S. \& JAIME, V., 1997. Efecto de Tres Estrategias de Suplementación com Hierro y Ácido Fólico para el Control y Prevención de la Anemia Ferropriva en Mujueres Embarazadas. 4 Mayo $2002<$ http://www.bireme.br>.

FUJIMORI, E.; LAURENTI, D.; NUNEZ-DE-CASSANA, L. M.; OLIVEIRA, I. M. V. \& SZARFARC, S. C., 2000. Anemia e deficiência de ferro em gestantes adolescentes. Revista de Nutrição, 13:177-184.

FUJIMORI, E.; OLIVEIRA, I. M. V.; CASSANA, L. M. N. \& SZARFARC, S. C., 1999. Estado nutricional del hierro de gestantes, São Paulo, Brasil. Archivos Latinoamericanos de Nutrición, 49:8-12.

GOLDENBERG, R. L.; TAMURA, T.; NEGGERS, Y.; COPPER, R. L.; JOHNSTON, K. E.; DUBARD, M. B. \& HAUTH, J. C., 1995. The effect of zinc supplementation on pregnancy outcome. JAMA, 274:463-468.

HUNT, I. F.; MURPHY, N. J.; CLEAVER, A. E.; FARAJI, B.; SWENDSEID, M. E.; BROWDY, B. L.; COULSON, A. H.; CLARK, V. A. \& SETTLAGE, R. H., 1985. Zinc supplementation during pregnancy in lowincome teenagers of Mexico descent: Effects on selected blood constituents and progress and outcome of pregnancy. American Journal of Clinical Nutrition, 42:815-828.

HALL, M. H., 1976. The cause of the fall in serum folate in normal pregnancy. British Journal of $\mathrm{Ob}$ stetric and Gynaecology, 83:132-136.

HAMBIDGE, K. M. \& DROEGMULLER, W., 1974. Changes in plasma and hair concentrations of zinc, copper, chromium, and manganese during pregnancy. Obstetric and Gynecology, 44:666-672.

INSTITUTE OF MEDICINE, 2001. Dietary Referency Intakes for Vitamin A, Vitamin K, Arsenic, Boron, Chromium, Copper, Iodine, Iron, Manganese, Molybdenum, Nickel, Silicon, Vanadium, and Zinc. Washington, DC: National Academy Press.

KAISER, L. L.; TAMURA, T.; HALSTED, C. H. \& HURLEY, L. S., 1981. Intestinal and hepatic folate conjugase in zinc deficient rats. Federation Proceeding, 40:865.

KEEN, C. L. \& HURLEY, L. S., 1989. Zinc and reprodution: effects of deficiency on foetal and postnatal development. In: Zinc in Human Biology (C. V. Mills, org.), p. 388, London: Springer-Verlag.

KIRKSEY, A.; WACHS, T. D.; YUNIS, F.; SRINATH, U.; RAHMANIFAR, A.; McCABE, G. P.; GALAL, O. M.; HARRISON, G. G. \& JEROME, N. W., 1994. Relation of maternal zinc nutriture to pregnancy outcome and infant development in an Egyptian village. American Journal of Clinical Nutritrion, 60:782-792.
KNIGHT, E. M.; SPURLOCK, B. G.; EDWARDS, C. H.; JOHNSON, A. A.; OYEMADE, U. J.; COLE, O. J.; WEST, W. L.; MANNING, M.; JAMES, H.; LARYEA, H.; WESTNEY, O. E.; JONES, S. \& WESTNEY, L. S., 1994. Biochemical profile of Africa american women during three trimesters of pregnancy and at delivery. Journal of Nutrition, 124:943-953.

NICHOLS, J.; MORGAN, J. \& TAILOR, A., 1992. Postnatal depression and zinc status - A preliminary study. Journal of Nutrition Medical, 3:35.

NOETHER, G. E., 1980. Estatística. São Paulo: Guanabara Dois.

NOGUEIRA, N. N.; VAZ PARENTE, J.; MARREIRO, D. N. \& COZZOLINO, S. M. F., 2001. Utilização de diferentes concentrações de ferro por adolescentes grávidas suplementadas também com zinco e ácido fólico. Archivos Latinoamericanos de Nutrición, 51:225-229.

SALZANO A. C.; BATISTA FILHO, M.; FLORES, H. \& CALADO, C. L. A., 1980. Prevalência de anemia no ciclo gestacional em dois Estados do Nordeste brasileiro, Pernambuco e Paraíba. Revista Brasileira de Pesquisas Médicas e Biológicas, 13:211214.

SZARFARC, S. C., 1983. Prevalência de Anemias Nutricionais entre Gestantes Matriculadas em Centros de Saúde do Estado de São Paulo. Tese de Livre Docência, São Paulo: Faculdade de Saúde Pública, Universidade de São Paulo.

SWANSON, C. A. \& KING, J. C., 1983. Reduced serum zinc concentration during pregnancy. Obstetric and Gynecology, 62:313.

TUTTLE, S.; AGGETT, P. J.; CAMPBELL, D. \& MACGILLIVRAY, I., 1985. Zinc and cooper nutrition in human pregnancy: A longitudinal study in normal primigravidae and in primigravidae ar risk of delivering a group relarded baby. American Journal of Clinical Nutrition, 41:1032-1041.

TAMURA, T.; SHANE, B.; BAER, M. T.; KING, J. C.; MARGEN, S. \& STOKSTAD, R., 1978. Absorption of mono and polyglutamil folates in zinc - Depleted man. American Journal of Clinical Nutrition, 31:1984-1987.

ZAVALETA, N.; CAULFIELD, L. E. \& GARCIA, T., 2000. Changes in iron status during pregnancy in peruvian women receiving prenatal iron and folic acid supplements with or without zinc. American Journal of Clinical Nutrition, 71:956-961.

ZIMMERMAN, A. W.; DUNHAM, B. S.; NOCHIMSON, D. J.; KAPLAN, B. M.; CLIVE, J. M. \& KUNKEL, S. L., 1984. Zinc transport in pregnancy. American Journal of Obstetric and Gynecology, 149:523-529.

Recebido em 19 de julho de 2001

Versão final reapresentada em 4 de junho de 2002

Aprovado em 30 de julho de 2002 